\title{
Application of a multiscale constitutive framework to real gas turbine components
}

\author{
T. Tinga ${ }^{1, a}$, W.A.M. Brekelmans ${ }^{2, b}$ and M.G.D. Geers ${ }^{2, c}$ \\ ${ }^{1}$ Netherlands Defence Academy, PO Box 10000, 1780 CA Den Helder, The Netherlands \\ ${ }^{2}$ Eindhoven University of Technology, PO Box 513, 5600 MB, Eindhoven, The Netherlands \\ at.tinga@nlda.nl, bw.a.m.brekelmans@tue.nl, cm.g.d.geers@tue.nl
}

Key words: Nickel Base Alloy, Degradation, Modelling, Microstructure, Mechanical properties.

\begin{abstract}
A multiscale constitutive framework for Ni-base superalloys has been developed, in which an efficient unit cell is adopted to describe the $\gamma / \gamma^{\prime}$ microstructure morphology. The framework enables the prediction of the deformation and the creep and fatigue damage accumulation in CMSX-4 for a range of temperatures and stress levels. Moreover, the material microstructural degradation due to rafting and isotropic coarsening can be simulated, and the effects of this degradation on the alloy mechanical response can be quantified. The present paper focuses on the application of the model to real gas turbine components. A high pressure turbine blade finite element model is used to demonstrate the computational efficiency of the multiscale framework. Moreover, the location of critical regions and the life time are shown to differ from the results obtained from classical models that neglect the microstructure evolution.
\end{abstract}

\section{Introduction}

Gas turbines are extensively used for power generation and for the propulsion of aircraft and vessels. Their most severely loaded parts, the turbine rotor blades, are manufactured from single crystal nickel-base superalloys. The superior high temperature behaviour of these materials is attributed to the two-phase composite microstructure consisting of a $\gamma$-matrix (Ni) containing a large volume fraction of $\gamma^{\prime}$-particles $\left(\mathrm{Ni}_{3} \mathrm{Al}\right)$. During service, the initially cuboidal precipitates evolve to elongated plates through a diffusion-based process called rafting. To include this deterioration process in the life assessment of gas turbine components, models must be available that include the specific microstructural dimensions and the changes therein.

Therefore, facilitated by the National Aerospace Laboratory NLR, a multiscale framework has been developed [1-4] that incorporates the effect of microstructure degradation on the mechanical response and damage accumulation. In the next sections, firstly the framework will be discussed, including the constitutive models for the matrix [1] and precipitate [4] phases, the developed damage model [2] and the modeling of the microstructure degradation [3]. But the focus of the present paper is on the application of the model to real gas turbine components. The benefits of using the multiscale model will be demonstrated.

\section{Multiscale framework}

The developed multiscale model for the prediction of the superalloy mechanical behaviour covers several length scales [1], which is shown schematically in Figure 1a. The macroscopic length scale characterises the engineering level on which a finite element (FE) model is commonly used to solve the governing equilibrium problem. The mesoscopic length scale represents the level of the microstructure within a macroscopic material point. At this length scale the material is considered as a compound of two different phases: $\gamma^{\prime}$-precipitates embedded in a $\gamma$-matrix. Finally, the microscopic length scale reflects the crystallographic response of the individual material phases. The constitutive behaviour is defined on this level using a strain gradient crystal plasticity framework. 
On the material point level the Ni-base superalloy microstructure, consisting of $\gamma^{\prime}$-precipitates in a $\gamma$-matrix, is represented by a unit cell containing 16 regions (see Figure $1 \mathrm{~b}$ ): $1 \gamma^{\prime}$-precipitate region, $3 \gamma$-matrix channel regions $\left(\gamma_{\mathrm{j}}, j=1 \ldots 3\right)$ with different orientations and 12 interface regions $\left(\mathrm{I}_{\mathrm{k}}{ }^{\mathrm{m}}\right.$ and $\left.\mathrm{I}_{\mathrm{k}}{ }^{\mathrm{p}}, k=1 \ldots 6\right)$ containing the $\gamma / \gamma^{\prime}$-interfaces. A matrix and a precipitate region together form a bi-crystal located on each face of the $\gamma^{\prime}$-precipitate. The latter interface regions were included in the model, since the interface between the two different phases plays an important role in the mechanical behaviour of the material. In these regions, strain gradient induced back stresses develop as well as stresses originating from the lattice misfit between the two phases. The limited size of the unit cell and the micromechanical simplifications make the framework particularly efficient in a multiscale approach.

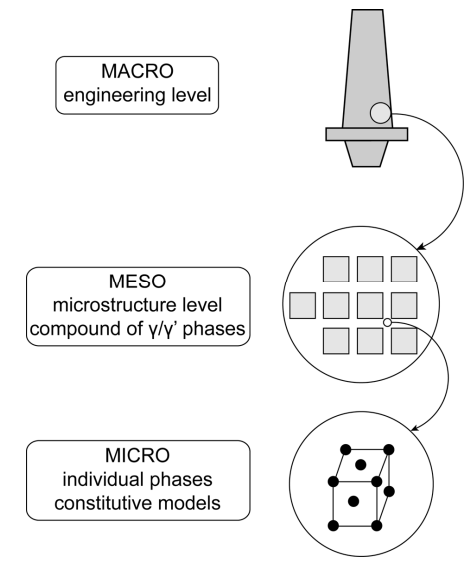

a)

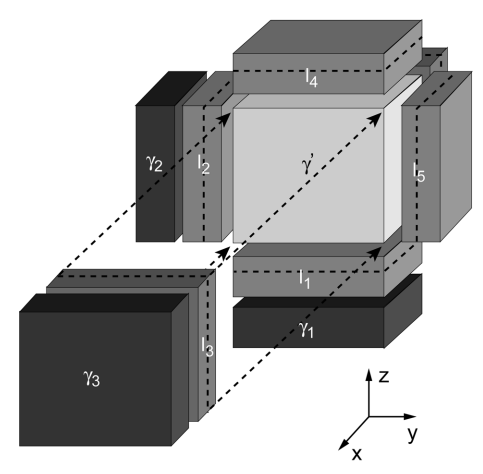

b)

Figure 1 Schematic overview of the model, showing (a) the multi-scale character and (b) the multi-phase unit cell, consisting of one precipitate $\left(\gamma^{\prime}\right)$, three matrix $\left(\gamma_{i}\right)$ and six double interface $\left(I_{i}\right)$ regions.

Matrix constitutive model. The matrix phase constitutive behaviour is simulated by using a nonlocal strain gradient crystal plasticity model [1]. The basic ingredient of the model is the relation between the slip rates $\dot{\gamma}^{\alpha}$ and the resolved shear stresses $\tau^{\alpha}$ for all the slip systems $\alpha$. The following formulation is used:

$$
\dot{\gamma}^{\alpha}=\dot{\gamma}_{0} F\left(\Omega^{\alpha}\right)\left\{\frac{\left|\tau_{e f f}^{\alpha}\right|}{s^{\alpha}}\right\}^{m}\left\{1-\exp \left(-\frac{\left|\tau_{e f f}^{\alpha}\right|}{\tau^{o r}}\right)\right\}^{n} \operatorname{sign}\left(\tau_{e f f}^{\alpha}\right)
$$

where $\tau^{o r}$ denotes the Orowan stress, $s^{\alpha}$ the actual slip resistance and $\tau_{\text {eff }}^{\alpha}$ the effective shear stress on slip system $\alpha$, obtained from the effective stress tensor $\boldsymbol{\sigma}_{\text {eff }}$ using the symmetric Schmid tensor. The factor $F$ is a cross slip factor that will be discussed below, while $\dot{\gamma}_{0}, m$ and $n$ are material constants.

The effective stress tensor is defined as the combination of the externally applied stress, the strain gradient induced back stress and the misfit stress. The formulation in equation (1) is an extended version of the slip law for a single phase FCC material [5]. For the present two-phase material an additional threshold term is added to account for the Orowan stress, which is the stress required to bow a dislocation line into the channel between two precipitates. This stress is inversely proportional to the spacing between two precipitates. As will be shown later, degradation of the microstructure changes the spacing between precipitates and therefore affects the Orowan stress and the slip rates. Finally, the slip resistance is a function of the total dislocation density, composed of the statistically stored dislocations (SSDs) and geometrically necessary dislocations (GNDs) [1]. 
Cross slip. The majority of the superalloy constitutive models available in literature contain, in addition to the octahedral systems, a set of cube slip systems to correctly simulate the material behaviour at orientations other than $<001>$. However, experiments show that the occurrence of macroscopic cube slip is quite exceptional. In the present model, the octahedral cross slip processes, that are physically responsible for the major part of the cube slip, are modelled and their effect is incorporated [4]. A parameter $\Omega^{\alpha}$ is introduced to quantify the amount of cross slip. It is defined as:

$$
\Omega^{\alpha}=\left\{\begin{array}{ccc}
\left|\frac{\tau_{e f f}^{\alpha_{1}}+\tau_{e f f}^{\alpha_{2}}}{\sigma_{e q}}\right| & \text { for } & \tau_{e f f}^{\alpha_{1}} \tau_{e f f}^{\alpha_{2}} \geq 0 \\
0 & \text { for } & \tau_{e f f}^{\alpha_{1}} \tau_{e f f}^{\alpha_{2}}<0
\end{array}\right.
$$

A higher value of $\Omega^{\alpha}$ indicates a more favourable condition for cross slip. Therefore, a cross slip factor $F$ based on the value of $\Omega^{\alpha}$ is incorporated to enhance the slip rate of the slip systems.

Precipitate constitutive model. The major part of plastic deformation is accommodated by the matrix phase, where dislocations are moving through relatively narrow channels. When encountering a precipitate, a loop is created around the particle, enabling the dislocation to continue its motion on the slip plane behind the precipitate. This is illustrated in Figure 2a. The dislocation loop around the precipitate may disappear by two different mechanisms, both involving inelastic deformation of the precipitate.

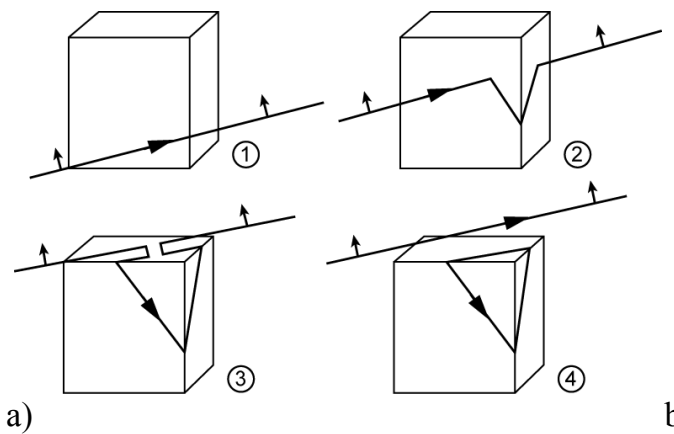

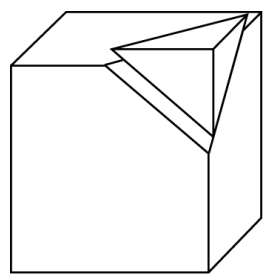

b)

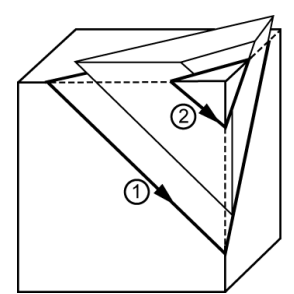

c)

Figure 2 a) Illustration of a moving matrix dislocation line encountering a precipitate (1) and creating a loop around the particle (2-4); b) Schematic representation of particle shearing and c) recovery climb.

The first mechanism is particle shearing (Figure $2 b$ ), where a dislocation line moves through the precipitate. The second mechanism of precipitate deformation is climb or recovery (Figure 2c), where the dislocation climbs around the precipitate. The latter process is thermally induced and involves diffusional processes and is therefore much slower than the previous mechanism. The mechanisms of precipitate shearing and recovery climb have been incorporated in the model [4].

Damage model. In addition to the model describing the material deformation, as discussed in the previous section, also a damage model has been developed [2] that integrates time-dependent and cyclic damage into a generally applicable time-incremental damage rule. This rule is based on the underlying physical mechanisms of both cyclic and time-dependent damage evolution. The formation and growth of micro voids in the material play a prominent role in damage accumulation under creep conditions [6, 7]. These voids, as well as casting pores, elongate and eventually coalesce just prior to failure during creep tests. This time-dependent part of the damage rule is based on the model proposed by Levkovitch et al. $[8,9]$.

On the micro-mechanical level, fatigue damage is attributed to irreversibility in the slip mechanism [10, 11]: upon load reversal the dislocations accommodating the plastic deformation should glide in the opposite direction. However, part of the dislocations is immobilized by reactions with other segments or due to cross-slip processes and cannot move backward, which means that other planes have to take over. In this way, the immobilization process gradually decreases the deformability of the material, which means that damage is accumulated. 
This mechanism is incorporated in the model by introducing a criterion based on the Orowan stress to detect slip reversal on the microscopic level. Also the effects of the magnitude of the cycle, temperature and the interaction with the time-dependent damage accumulation are incorporated in the model.

Microstructure degradation. The rafting and coarsening processes are modelled by defining evolution equations for several of the microstructural dimensions [3], as is illustrated in Figure 3. The phenomenological relation for the horizontal channel widening rate $\dot{h}$ during vertical tensile loading as derived by Epishin et al. [12] was taken as the point of departure for the present model. However, the relation was extended to be applicable for a general multiaxial stress state. The evolution rate of the precipitate size $\left(\dot{L}_{\mathrm{i}}\right)$ is then given by:

$$
\dot{L}_{\mathrm{i}}(T, \sigma)=-\frac{3}{2} L_{\mathrm{i}}\left[\frac{\sigma_{\mathrm{ii}}^{\mathrm{d}}}{\sigma_{\mathrm{VM}}+\varepsilon}\right] \frac{A^{*}}{L} \exp \left[-\frac{Q-\sigma_{\mathrm{VM}} U(T)}{R T}\right]
$$

where $\sigma_{\mathrm{ii}}$ and $\sigma_{\mathrm{VM}}$ are the deviatoric and Von Mises stress, respectively. The function $\mathrm{U}(\mathrm{T})$ defines the temperature dependence, whereas $\varepsilon, A^{*}$ and $\mathrm{Q}$ are model parameters. In addition to the rafting, a formulation for the coarsening process is derived, quantifying the evolution of the microstructure periodicity $\lambda$ (see Figure 3 ). Since the microstructural dimensions are already part of the model, the effect of the microstructure degradation on the mechanical response of the material is automatically obtained.

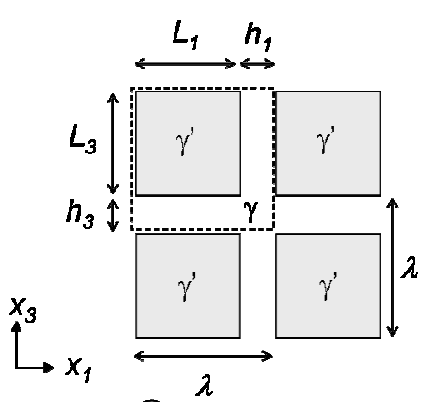

(1)

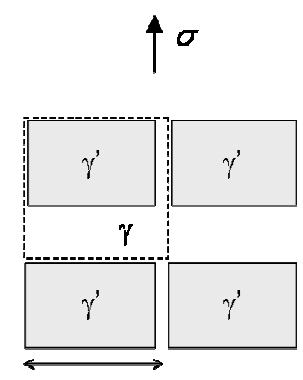

(2)

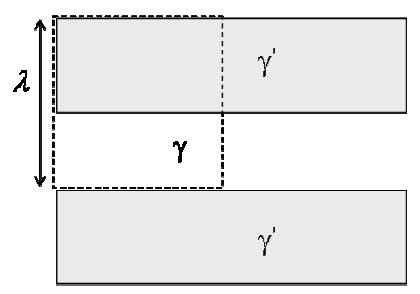

(3)

Figure 3 Schematic representation of the changes in microstructural dimensions at three different stages of the degradation process: 1) initial state; 2) microstructure in a rafted state; 3) fully rafted microstructure after a certain amount of coarsening.

\section{Application}

The framework capabilities are demonstrated by applying the model to smooth CMSX-4 specimens that have been tested within the COST538 Action. The predicted tensile and cyclic response are compared to the experimental results. Finally, the computational efficiency is illustrated by applying the model in a gas turbine blade finite element analysis.

Smooth specimens. Figure 4 shows the comparison of simulated and experimentally obtained tensile curves for specimens with different degrees of degradation (characterized by the increasing channel width $h$ ). The model appears to simulate these tests quite well. Also the results of a cyclic test on virgin material is compared to the simulated curve, where again the experimental results are reproduced quite well.

Gas turbine blades. To clearly emphasize the computational efficiency of the present framework and to demonstrate the multi-scale capabilities, the proposed multi-scale model was applied to a finite element model of an aero-engine low pressure turbine blade. The model was created at the National Aerospace Laboratory NLR [13] and contains 9150 nodes. The discussed 
unit cell model was implemented in a user-subroutine of the commercial finite element code MSC.Marc. The temperature distribution in the blade has been determined in a thermal FE analysis, including the effect of film cooling [14]. The combination of the thermal stresses and the centrifugal load $(14.000 \mathrm{rpm})$ yields the total stress distribution. Then the microstructure degradation in the turbine blade is simulated by performing a creep analysis for 1000 hours.
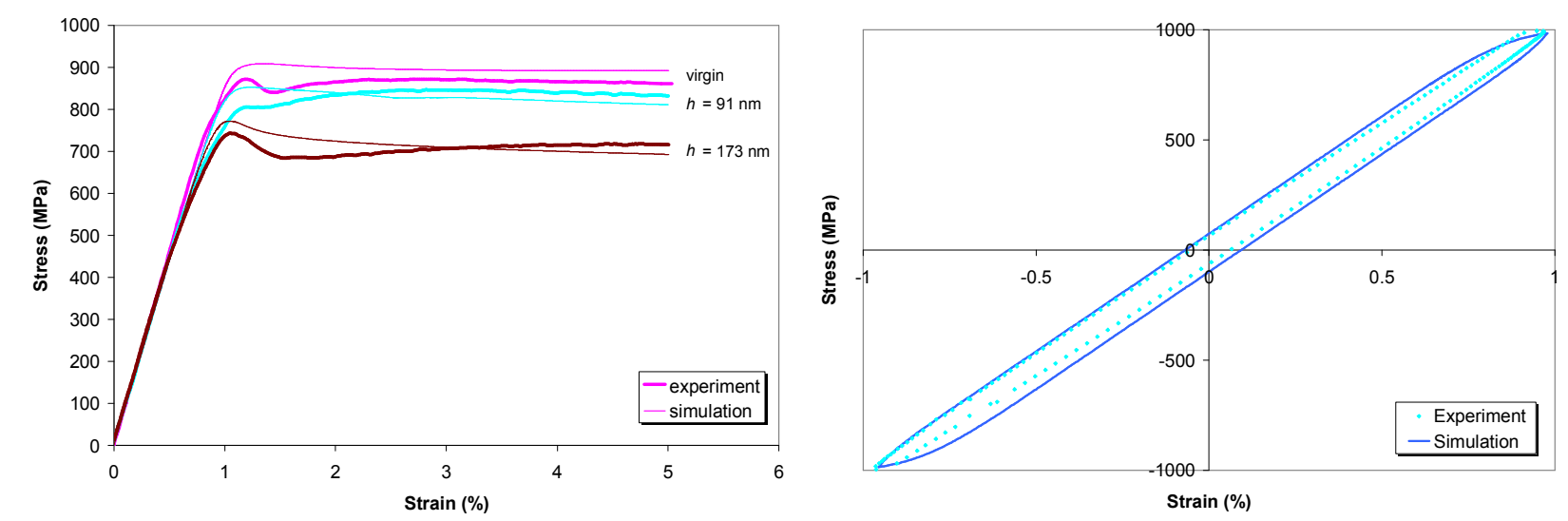

Figure 4 a) Simulated tensile tests $\left(950{ }^{\circ} \mathrm{C}\right.$ and $\left.10^{-3} \mathrm{~s}^{-1}\right)$ compared to experimental results for different degrees of degradation; b) Simulated and experimentally determined cyclic test (virgin, 1.93\% applied strain range).

The kinetics of the microstructure degradation depends on both the temperature and the stress state. Obviously, in a real component, the precise combination of temperature and stress state is different in almost any material point, resulting in a large variety of degraded microstructures over the turbine blade. The whole range of microstructures can be sorted into four classes, as is also shown in Figure 5a: non-degraded, partly rafted, fully rafted or isotropically coarsened.

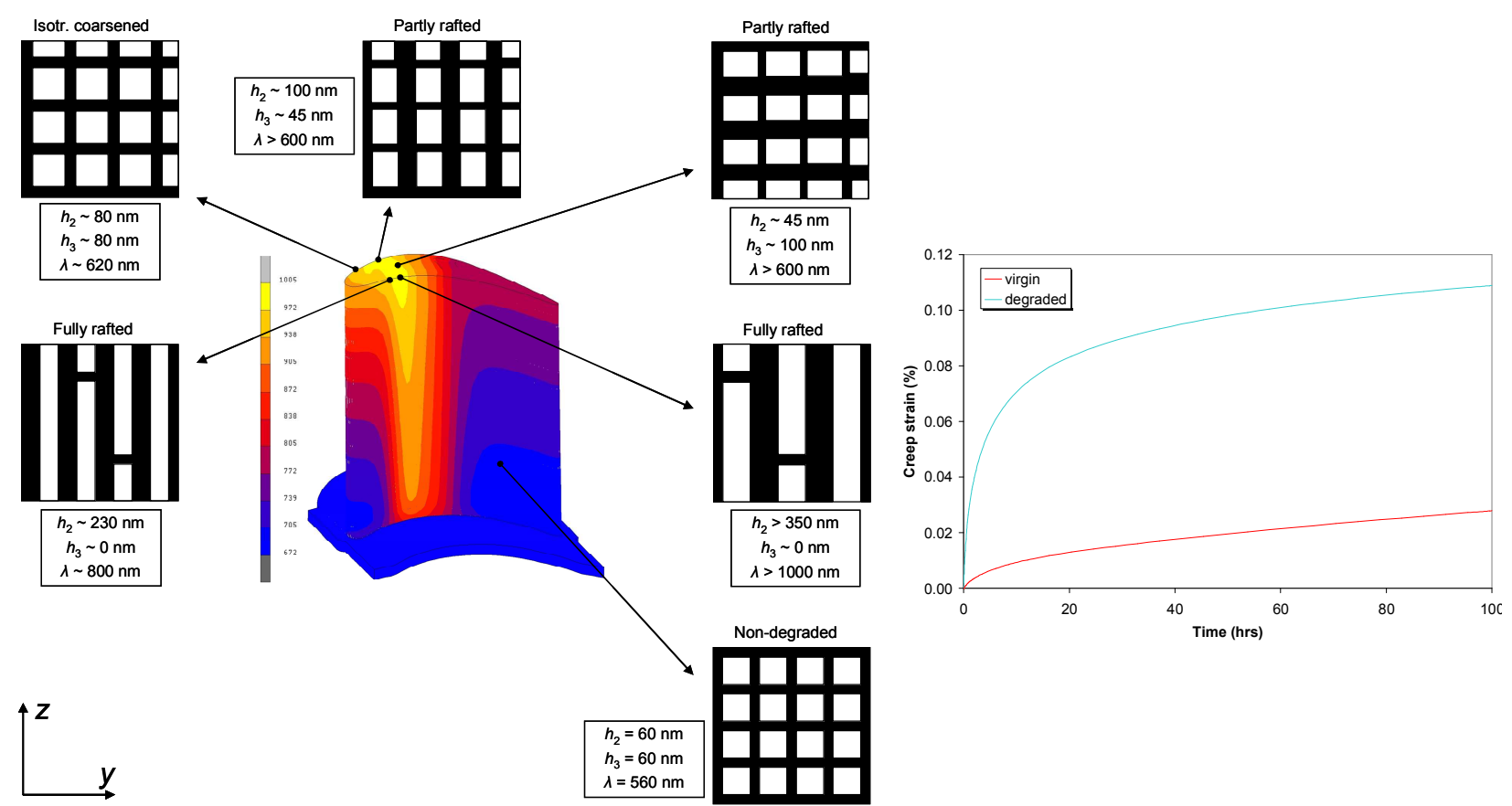

Figure 5 a) Overview of degraded microstructures observed after a simulation of degradation in a turbine blade under service conditions for 1000 hours. The contours indicate the blade temperature distribution. b) Comparison of the equivalent creep strain evolution in a virgin and a degraded blade for a specific location at the blade tip. 
These observations can be compared to the results presented by Epishin et al. [12]. They experimentally examined the microstructure at three different locations in an ex-service CMSX-4 (uncooled) turbine blade. The almost virgin microstructure at the blade platform and the varying orientation of the rafts at the tip region nicely correspond to the results shown in Figure 5 . The fully rafted microstructure at the blade centre was not observed here for two reasons: the simulated duration of $1000 \mathrm{hrs}$ is much shorter than the $12.700 \mathrm{hrs}$ in [12], and the internal blade cooling yields much lower temperatures in the centre region of the blade, which reduces the degradation rate there. These results clearly demonstrate that the typical case of a material loaded uniaxially in radial direction, which is mostly studied in literature, is not representative for most of the locations in this real component. That is especially due to the fact that the present blade is internally cooled, resulting in thermal stresses that cause the stress state to be multiaxial.

Concurrent with the microstructure degradation, also the creep deformation of the blade is calculated. The results indicate that the high creep strain regions at the suction side of the blade are hardly affected by the degradation. Moreover, the maximum value of the creep strain, located at the inner surface of the blade in one of the cooling channels, has decreased slightly from $1.30 \cdot 10^{-3} \mathrm{~m} / \mathrm{m}$ in the virgin blade to $1.27 \cdot 10^{-3} \mathrm{~m} / \mathrm{m}$ in the degraded blade. However, the equivalent creep strain near the tip at the pressure side is much higher in the degraded blade than in the virgin blade. The evolution of the creep strain at this location is plotted in Figure $5 b$ for both the virgin and the degraded blade, showing a significant increase in the creep rate.

These results demonstrate that the effect of degradation on the overall creep deformation is rather limited for this specific component, so the blade elongation is hardly affected by the degradation. However, local creep strain rates, especially in the hot regions of the blade, considerably increase after degradation. Neglecting the effects of rafting and coarsening in the numerical analysis would thus lead to unconservative life predictions. This clearly demonstrates the benefit of a model that includes the effects of degradation.

\section{Conclusions}

The present paper has demonstrated that constitutive models including the effects of microstructure degradation are required to accurately model the mechanical response of gas turbine components. Moreover, it has been shown that the computational efficiency of the applied multiscale framework is such that analyses on real components are feasible.

\section{References}

[1] Tinga, T., W.A.M. Brekelmans, and M.G.D. Geers, Phil. Mag. 2008. 88(30-32): p. 37933825.

[2] Tinga, T., W.A.M. Brekelmans, and M.G.D. Geers, Mat. Sci. Engng A 2009. 508: p. 200-208.

[3] Tinga, T., W.A.M. Brekelmans, and M.G.D. Geers, Comp. Mat. Sci. 2009. 47: p. 471-481.

[4] Tinga, T., W.A.M. Brekelmans, and M.G.D. Geers, Mod. Sim. Mat. Sci. Engng 2010. 18(015005): p. 1-31.

[5] Evers, L.P., W.A.M. Brekelmans, and M.G.D. Geers, J. Mech. Phys. Sol. 2004. 52: p. 23792401.

[6] Moss, S.J., G.A. Webster, and E. Fleury, Metall. Mat. Trans. A 1996. 27A: p. 829-837.

[7] Reed, R.C., N. Matan, and others., Acta Mat. 1999. 47(12): p. 3367-3381.

[8] Levkovitch, V., R. Sievert, and B. Svendsen. 2003. Berlin: D. Verb. Mat. fors. prüf.

[9] Levkovitch, V., R. Sievert, and B. Svendsen, Int. J. Fatigue 2006. 28 p. 1791-1802.

[10] Shyam, A. and W.W. Milligan, Acta Mat. 2005. 53: p. 835-844.

[11] Fedelich, B., Int. J. Plast. 2002. 18: p. 1-49.

[12] Epishin, A., et al. 2008. Champion, Pennsylvania, USA: Min. Met. Mat. Soc.

[13] Tinga, T., W.B. De Wolf, and others. 2001. Manchester, UK: NATO RTO.

[14] De Wolf, W.B., S. Woldendorp, and T. Tinga. 2001. Loen, Norway: NATO RTO. 